\title{
Development of a Molecularly Imprinted Polymer (MIP) for the Recovery of Lactoferrin
}

\author{
Méndez-Palacios I. ${ }^{1,2}$, López-Luna A. ${ }^{2}$, Bárzana E. ${ }^{2}$, Jiménez-Guzmán J. ${ }^{1}$, García-Garibay \\ M. ${ }^{1}$ \\ ${ }^{1}$ Departamento de Biotecnología, Universidad Autónoma Metropolitana, Iztapalapa, A. P. 55- \\ 535, Mexico, City. Mexico. Tel: +(52)(55)5804-4720 Fax: +(52)(55)5804-4712 E-mail: \\ jmgg@xanum.uam.mx \\ ${ }^{2}$ Departamento de Alimentos y Biotecnología, Facultad de Química, UNAM, 04510, Mexico \\ City, Mexico
}

\begin{abstract}
Lactoferrin (LF), the most valuable protein of whey, was recovered by molecular imprinting using either vinylpiridin alone (MIP1) or mixed with metacrilic acid (MIP2) as functional monomers, and etylenglycol dimetacrilate was selected as crosslinker under different polymerization conditions. In order to create the specific cavity pure LF was used as template. A control was prepared for every MIP using the same monomers and polymerization conditions but without the template. The polymers obtained were tested against a protein mixture containing LF, and the recovery efficiency was calculated determining the diminution of LF from the supernatant by titration of the iron of the LF, as well as native PAGE to determine the protein composition of the supernatants. The only polymer which was able to specifically bind LF was MIP1 (vinylpiridin alone). Native PAGE showed that when MIP1 was used against the mixture of proteins, LF diminished with respect to the others. Measuring the proportions of the different proteins in the mixture, it was demonstrated that before the separation with MIP1, LF represented $82 \%$ of the total protein content, and diminished to $62 \%$ after the exposure to MIP1, suggesting that LF was specifically sorbed by this polymer. The retention efficiency of the polymer showed that MIP1 retained $27 \%$ of the total LF content, while its control retained only $1.6 \%$, demonstrating that the retention of the protein is not due to unspecific adsorption in the polymer, but rather to a selective retention in the cavity formed by the template.
\end{abstract}

Keywords: Lactoferrin, whey proteins, molecular imprinting

\section{Introduction}

Lactoferrin (LF) has become in recent years one of the most valuable proteins of whey due to its nutraceutical properties. It is a glycoprotein composed by 689 aminoacids and with a molecular mass of $80 \mathrm{kDa}$, which is found in milk in a low concentration (about $0.1 \mathrm{~g} / \mathrm{l}$ ). It has a great capacity to bind $\mathrm{Fe}^{3+}$, containing two molecules of this metal per protein chain.

Recently, lactoferrin has aroused great interest due to its biological and nutraceutical properties such as: bacteriostasis/bactericidal effect, immunomodulating properties, antinflammatory effect, growth stimulating properties, antiviral activity and enhancer of iron absorption (Lönnerdal 2003; Korhonen and Marnila 2003). Beyond the scientific interest on lactoferrin due to biological and health promoting properties, the commercial interest on this protein has been greatly increased in recent years leading to the search of proper methodologies for its isolation and purification from whey. However, current techniques are little specific, then commercial products are just lactoferrin enriched mixtures of several whey proteins.

Molecular imprinting is a method for preparing synthetic materials able to mimic the molecular recognition phenomena present in living systems. It consists in the selection of a 
template molecule which is later associated with some functional monomers through noncovalent binds; then, a polymerization around the template-monomer complex is conducted resulting in a molecularly imprinted polymer (MIP) which has a cavity that recognizes the template molecule, allowing to capture it specifically separating it from a complex mixture. Up to date, this technique has mainly been used for the separation of low molecular weight molecules (Piletsky et al, 2001). The aim of this work was to develop a molecularly imprinted polymer which allows the selective recovery of lactoferrin.

\section{Materials and Methods}

Vinylpiridin (Aldrich) alone (MIP1) or mixed with metacrilic acid (Mallinckrodt) (MIP2) was used as functional monomer, and etylenglycol dimetacrilate (Aldrich) was used as crosslinker. Potassium persulfate was used as catalyst to start the polymerization. In order to create the specific cavity pure LF was used as template. LF was purified from a commercial preparation (Bioferrin 2000, Glanbia Nutritionals, Monroe WI USA) by isoelctrofocusing (Rotofor, BioRad Laboratories, Hercules CA USA) and its purity was determined by native polyacrilamide gel electrophoresis (native-PAGE). A control was prepared for every MIP (B1 and B2) using the same monomers and polymerization condition but without the template. Polymerization conditions were $16 \mathrm{~h}$ at $60^{\circ} \mathrm{C}$; the reaction mixture was bubbled with nitrogen for $5 \mathrm{~min}$ at the beginning of the reaction in order to displace oxygen.

The molecular imprinted polymers were washed with a mixture of methanol and acetic acid during $1 \mathrm{~h}$ to remove the template.

The polymers obtained were tested against a protein mixture containing LF, and the recovery efficiency was calculated determining the diminution of LF from the supernatant by titration of the iron of the LF with KSCN, as well as by native-PAGE to determine the protein composition of the supernatants. PAGE was done by means of a Mini-Protean III equipment, and the concentration of proteins on gels were determined by a Gel Doc 1000 (BioRad, Hercules CA USA).

Every experiment was performed three times and results were analyzed by ANOVA and by Tukey-Kramer test using the Statistica software for Windows V 5.0.

\section{Results and Discussion}

\section{LF purification}

After the isoelctrofocusing purification of the commercial lactoferrin preparation, the sample was analyzed by native-PAGE $(\mathrm{T}=10 \%)$. Figure 1 shows the gel in which the commercial product and the pure sample of LF that was used as template purification efficiency can be observed by comparing lanes with LF before and after purification.

It can be seen that in the commercial product LF is contaminated with several other proteins; according to their molecular weight it is likely that these proteins could be mainly immunoglobulins and bovine serum albumin. After isoelectrofocusing purification, a pure sample of LF was obtained which was used as template for the MIP production. The calculated molecular mass for pure LF according to the PAGE analysis was $86.6 \mathrm{kDa}$, and it corresponded to the fraction with $\mathrm{pI}=8.0$; both characteristics are very close to those reported for LF (80 kDa and 8.7 respectively) (Lönnerdal 2003). 


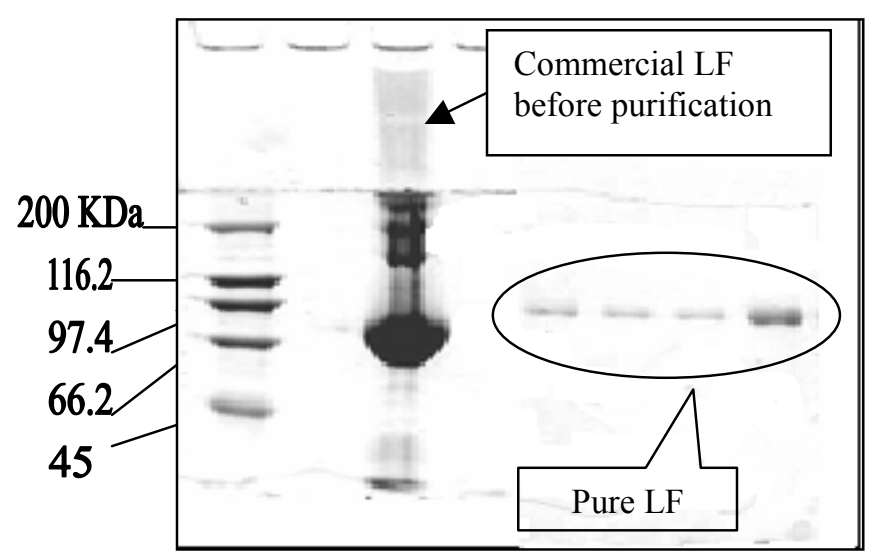

Figure 1. Native electrophoresis on polyacrilamide gel $(\mathrm{T}=10 \%)$ stained with Coomassie Blue.

\section{Production of the molecularly imprinted polymer}

Two polymers were produced: one using vinylpiridin alone (MIP1) as functional monomer, and the other using a mixture of vinylpiridin and metacrilic acid (MIP2). The corresponding controls (B1 and B2) were synthesized under the same conditions but without template.

After removing the template from the polymers, they were exposed to the commercial LF product and allowed to interact with it for $1 \mathrm{~h}$. After that, the polymer was centrifuged and the protein concentration of the supernatant was measured in order to determine the amount of protein which had been sorbed in the MIP (Figure 2).

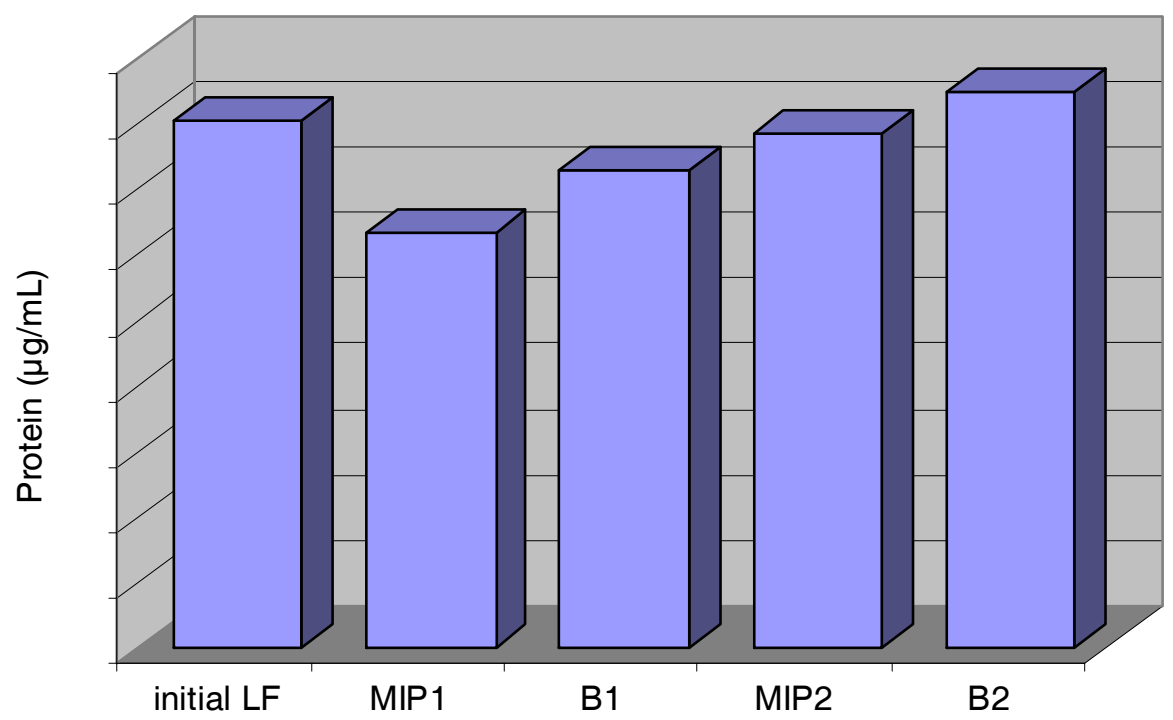

Figure 2. Protein concentration determined by Lowry in the supernatants after exposure to the MIPs and their controls (B).

Figure 2 shows that protein concentration diminished in every MIP with respect to its own control, but only in the case of MIP1 was this reduction significant $(\alpha=0.006)$ and high enough to be considered promising for LF recovery. 
Native-PAGE was used to evaluate the specificity of MIP1 to bind LF, showing that when used against the protein mixture it diminished LF concentration with respect to the other proteins (Figures 3 and 4). Measuring the proportions of the different proteins in the mixture, it was demonstrated that before the separation with MIP1, LF represented $82 \%$ of the total protein content, and diminished to $62 \%$ after the exposure to MIP1, suggesting that LF was specifically sorbed by this polymer.

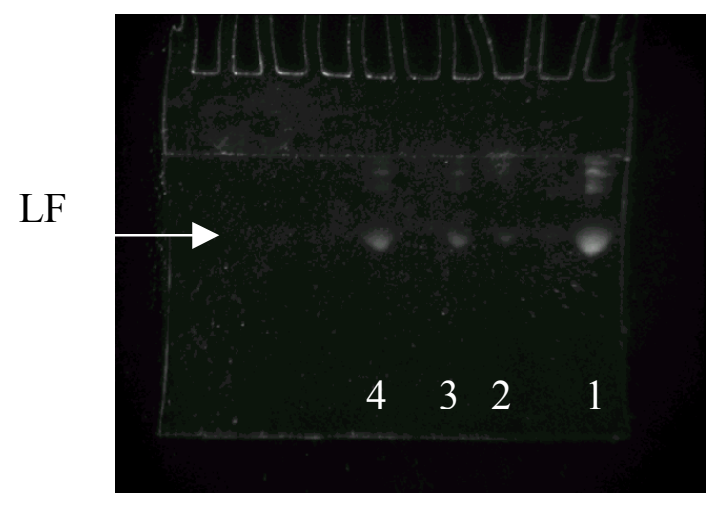

Figure 3. Native-PAGE of the supernatants after separation with MIP1. Lanes: 1.- Complex mixture of proteins: 2 and 3.- Supernatant of MIP1: 4.- Supernatant of the control

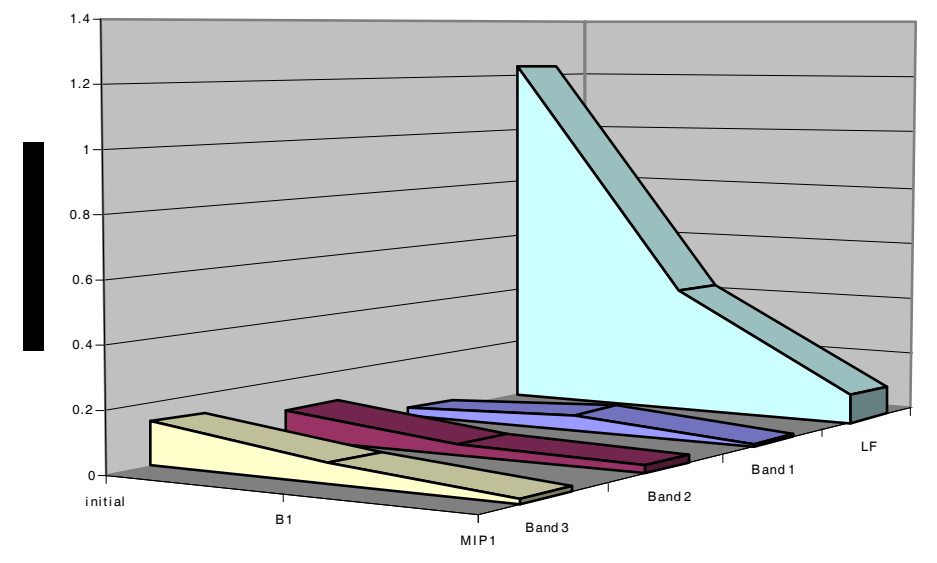

Figure 4. Protein retention in the MIP and its control measured from the Native-PAGE.

For better accuracy LF was measured in the supernatants by titrating its iron content with KSCN. Results confirmed that MIP1 retained $27 \%$ of the total LF content, while its control retained only $1.6 \%$ (Figure 5), demonstrating that the retention of the protein is not due to an unspecific adsorption in the polymer, but rather to a selective retention in the cavity formed by the template. 


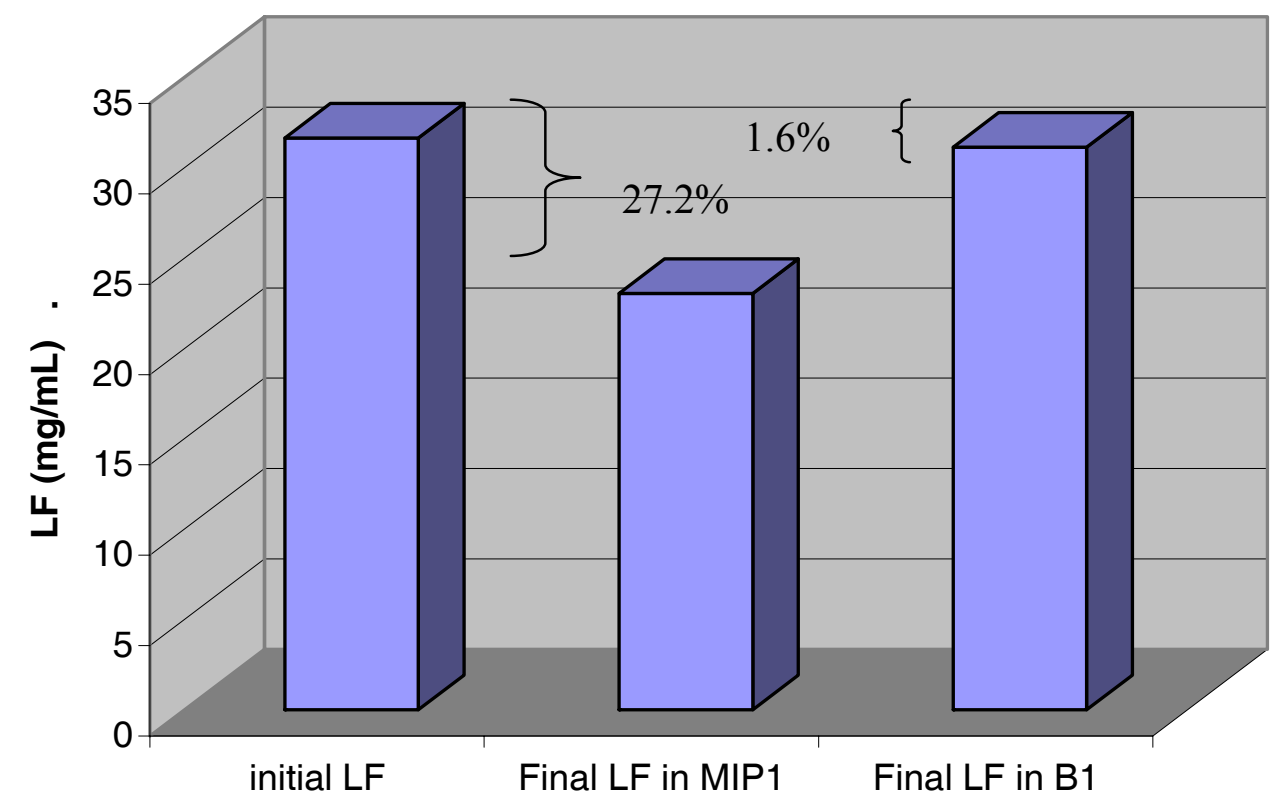

Figure 5. LF retained in MIP1 and its control determined by iron titration.

The system remains yet to be optimized, but the retention efficiency obtained so far suggests that molecular imprinting could be an interesting technology for the purification of valuable proteins from sources such as whey.

\section{Literatura cited}

- Korhonen H., Marnila P., (2003), Lactoferrin. In Encyclopaedia of Dairy Sciences, Roginski H., Fucquay J., and Fox, P.F, editors., Elsevier Science Academic Press, EUA.

- Lönnerdal B., (2003), Lactoferrin. In Advanced Dairy Chemistry, Vol. 1 Proteins, third edition Part A. Fox P.F. and McSweeney P.L.H. editors. Kluwer Academic/Plenum Publishers, New York.

- Piletsky S., Alcock S., Turner A., (2001) Molecular Imprinting: At the edge of the third millennium. Trends Biotechnol. 19, 9-12. 